\title{
Study on Heat Transfer of Composites Reinforced by Nanopaper
}

\author{
Aying Zhang ${ }^{1, a,}{ }^{*}$, Zhenghong Li ${ }^{2, b}$ \\ ${ }^{1}$ Harbin University, 150086 Harbin, China \\ ${ }^{2}$ Harbin Institute of Technology, 150001 Harbin, China \\ a,*zaying@sina.com, b273662999@qq.com
}

Keywords: Heat transfer, Simulation, Nanopaper, Composites.

\begin{abstract}
FLUENT was used to analyze how the bending cycles of nanopaper affects the temperature distribution of composites reinforced by pulse bending nanopaper with different bending cycles during heating process. The temperature distribution of the polymer composites reinforced by pulse bending nanopaper along the section $\mathrm{z}=0$ is more uniform with the increase of bending cycles of nanopaper. The difference of the maximum and minimum temperature of the composites increases linearly with the increase of the heating power. The difference of the maximum and minimum temperature of the composite decreases with the increase of the bending cycles of nanopaper, which indicates that the temperature distribution of the composite is more uniform.
\end{abstract}

\section{Introduction}

Carbon nanotubes (CNTs) have exceptionally good mechanical properties and thermal conductivity $[1,2]$. CNTs have some advantages beneficial for practical applications, including their excellent electrical, chemical, thermal and mechanical properties, and corrosion resistance to many severe environments. Due to their excellent advantages, CNTs were frequently mixed with polymers to enhance their thermal conducting performance [3]. However, manufacturing CNT-reinforced composites with uniform tube dispersion and large CNT loading is a great challenge [4]. CNT-buckypaper based composites can overcome part of the above-mentioned critical issues [5].

Previous research on the properties of buckypaper and buckypaper/polymer composites has mostly been concentrated on their mechanical properties and conductive properties. In this work, the thermal responses of the polymer composites reinforced by flat, sinusoidal and bent buckypaper were systematically studied and the heating mechanisms of multiple-field coupling were identified.

\section{Numerical model}

The heating model of the polymer composite reinforced by the nanopaper is shown in Figure 1. As shown in Figure 1, heating the nanopaper is driven by a power source, and the cube region is the polymer matrix which is heated by the nanopaper. The temperature of the polymer matrix is increased due to the electro-heating of the nanopaper. The schematic diagram of the heating structure shows that the polymer matrix is heated by the nanopaper when the nanopaper is applied to an electrical source. $T, L$ and $w$ are the thickness, length and width of the polymer matrix, and $h, d$ and $A$ are the bent height, thickness and bent period of the nanopaper. The length, width, and the thickness of the heating model of the polymer composite reinforced by the flat and bent nanopaper are $60 \mathrm{~mm}, 50 \mathrm{~mm}$, $100 \mathrm{~mm}$, and respectively. The thickness of the flat and bent buckypaper is $10 \mathrm{~mm}$. The bent height and bent period of the bent buckypaper are $60 \mathrm{~mm}$ and $120 \mathrm{~mm}$.

The objective of this research is to analyze how the bending cycles of nanopaper affects the temperature distribution of composites reinforced by pulse bending nanopaper with different bending cycles during heating process. 


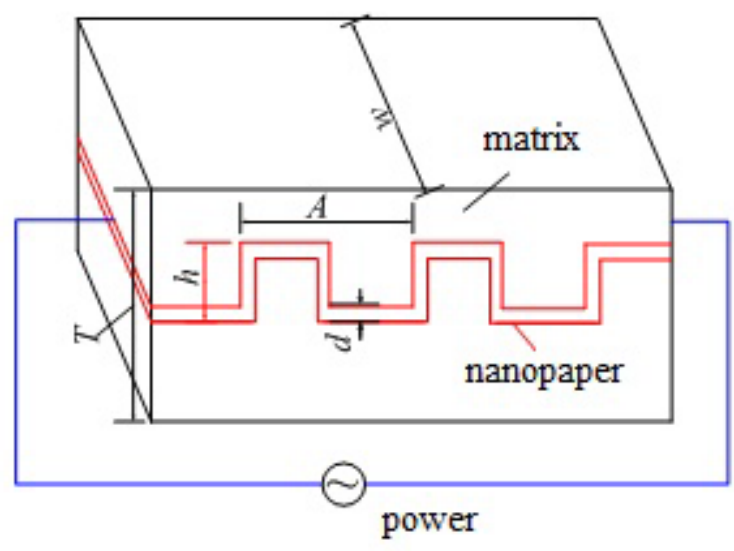

Fig. 1. Sketch diagram of heating experimental device

\section{Calculation Condition}

Boundary conditions were pre-seted as follow. The natural convection heat transfer coefficient was set to be $10 \mathrm{~W} /\left(\mathrm{m}^{2} \cdot \mathrm{K}\right)$. The ambient temperature was set to be $300 \mathrm{~K}$. The thermal conductivity of nanopaper was set to be $1.0 \mathrm{~W} /(\mathrm{m} \cdot \mathrm{K})$. The thermal conductivity of the polymer matrix were varied from $0.02,0.05,0.10$, and $0.20 \mathrm{~W} /(\mathrm{m} \cdot \mathrm{K})$. The specific heat capacity of nanopaper was set to be 1000 $\mathrm{J} /(\mathrm{kg} \bullet \mathrm{K})$. The density of nanopaper was set to be $500 \mathrm{~kg} / \mathrm{m}^{3}$. The thermal conductivity of the polymer matrix was set to be $0.1 \mathrm{~W} /(\mathrm{m} \bullet \mathrm{K})$. The specific heat capacity of the polymer matrix was set to be 1300 $\mathrm{J} /(\mathrm{kg} \cdot \mathrm{K})$. The density of the polymer matrix was set to be $1000 \mathrm{~kg} / \mathrm{m}^{3}$.

Figure 2 shows the geometry model of the polymer composites reinforced by pulse bending nanopaper.

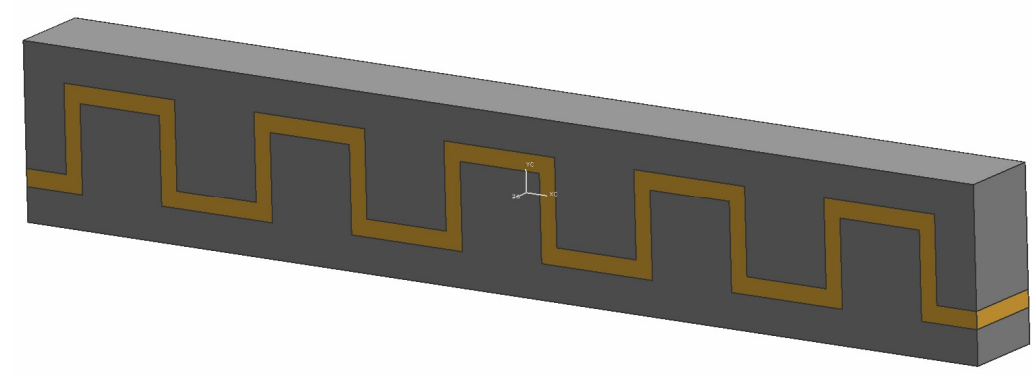

Fig. 2. Model of the polymer composites reinforced by pulse bending nanopaper

\section{Results and discussion}

The temperature of the polymer composites reinforced by pulse bending nanopaper at stable states along the section $\mathrm{z}=0$ under the volume internal heat source of $50000 \mathrm{~W} / \mathrm{m}^{3}$ is listed in Table 1 .

Table 1 Temperature of composites reinforced by pulse bending nanopaper along the sections $\mathrm{z}=0$

\begin{tabular}{ccc}
\hline $\mathrm{T}_{\max } / \mathrm{K}$ & $\mathrm{T}_{\min } / \mathrm{K}$ & $\mathrm{T}_{\text {ave }} / \mathrm{K}$ \\
\hline 343.99 & 302.37 & 329.47 \\
\hline
\end{tabular}

The temperature distribution of the polymer composites reinforced by pulse bending nanopaper at the stable states along the section $\mathrm{z}=0$ under the volume internal heat source of $50000 \mathrm{~W} / \mathrm{m}^{3}$ were 
calculated. The obtained temperature distribution maps are shown in Figures 3. Figures 3 shows that high temperature region appear in the vicinity of the nanopaper.

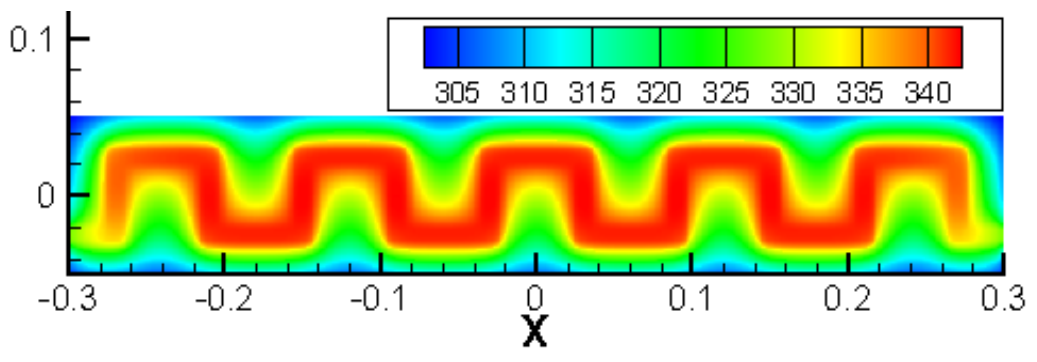

Fig. 3. Temperature distribution maps of composites reinforced by pulse bending nanopaper along the section $\mathrm{z}=0$ under the volume internal heat source of $50000 \mathrm{~W} / \mathrm{m}^{3}$

Figure 4 shows the temperature cloudy maps of composites reinforced by pulse bending nanopaper with different bending cycles along the section $\mathrm{z}=0$ under the heating power of $50 \mathrm{w}$. As shown in Figure 4, the temperature cloudy maps indicate that the temperature distribution of the polymer composites reinforced by pulse bending nanopaper along the section $\mathrm{z}=0$ is more uniform as the bending cycles of the nanopaper increase from $5 \mathrm{~A}$ to $8 \mathrm{~A}$ under the heating power of $50 \mathrm{~W}$.

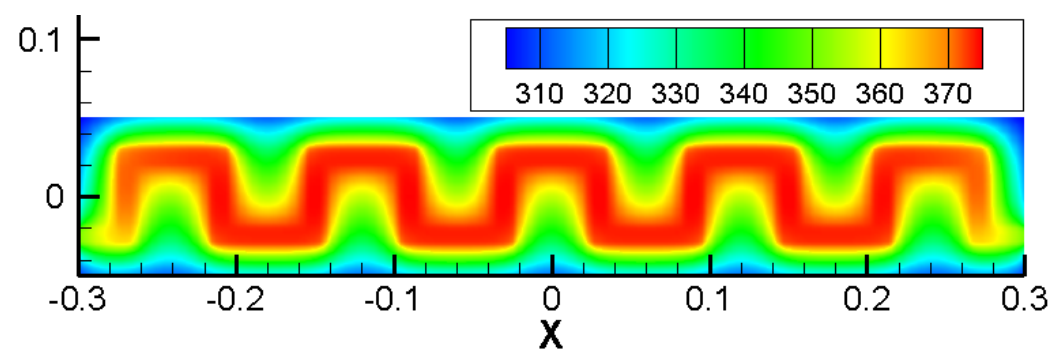

(a) $5 \mathrm{~A}$

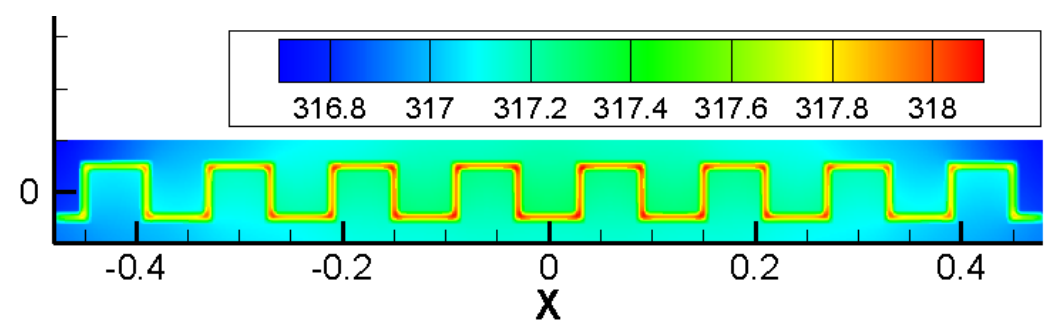

(b) $8 \mathrm{~A}$

Fig. 4. Temperature distribution of composites reinforced by pulse bending nanopaper with different bending cycles along the section $\mathrm{z}=0$ under the heating power of $50 \mathrm{w}$

Figure 5 shows the curve of temperature difference of composites reinforced by pulse bending nanopaper with different bending cycles along the section $\mathrm{z}=0$. As shown in Figure 5, the difference of the maximum and minimum temperature of the composite increases linearly with the increase of the heating power. Figure 5 shows that the difference of the maximum and minimum temperature of the composite decreases with the increase of the bending cycles of nanopaper, which indicate that the temperature distribution of the composite is more uniform. 


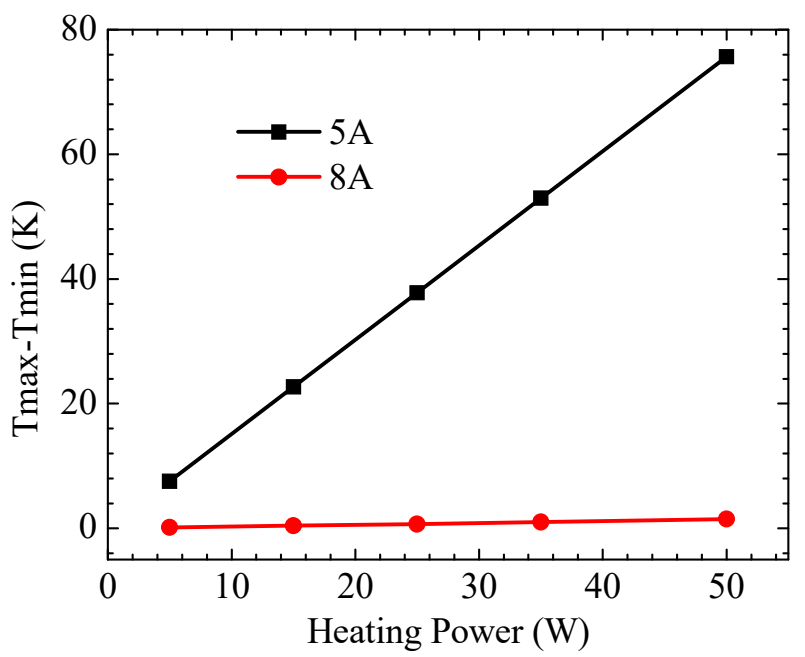

Fig. 5. Curve of temperature difference of composites reinforced by nanopaper with different bending cycles along the section $\mathrm{z}=0$

\section{Summary}

FLUENT was used to analyze how the bending cycles of nanopaper affects the temperature distribution of composites reinforced by pulse bending nanopaper with different bending cycles during heating process.

The temperature distribution of the polymer composites reinforced by pulse bending nanopaper along the section $\mathrm{z}=0$ is more uniform with the increase of bending cycles of nanopaper.

The difference of the maximum and minimum temperature of the composites increases linearly with the increase of the heating power. The difference of the maximum and minimum temperature of the composite decreases with the increase of the bending cycles of nanopaper, which indicates that the temperature distribution of the composite is more uniform.

\section{Acknowledgement}

This research were financially supported by Heilongjiang Natural Science Foundation (Grant No. E201454) and Heilongjiang Postdoctoral Scientific Research Developmental Fund (Grant No. LBH-Q16141).

\section{References}

[1] M. A. Osman and D. Srivastava, Temperature dependence of the thermal conductivity of single-wall carbon nanotubes, Nanotechnolgy, vol. 12, pp. 21-24, 2001.

[2] M. Fujii, X. Zhang, H. Xie and et al, Measuring the thermal conductivity of a single carbon nanotube, Physical Review Letters, vol. 95, pp. 065502, 2005.

[3] Q. Li, C. Liu, X. Wang and et al, Measuring the thermal conductivity of individual carbon nanotubes by the Raman shift method, Nanotechnology, vol. 20, pp. 5886-5890, 2009.

[4] A. A. Balandin, Thermal properties of graphene and nanostructured carbon materials, Nature Materials, vol. 10, pp. 569-581, 2011.

[5] E. Pop, D. Mann, Q. Wang and et al, Thermal conductance of an individual single-wall carbon nanotube above room temperature, Nano Letter, vol. 6, pp. 96-100, 2005. 